\title{
Absorbed fraction and dose conversion coefficients of alpha particles for radon dosimetry
}

\author{
D Nikezic ${ }^{1,2}, K_{\text {N Yu}}{ }^{1}$ and D Vucic ${ }^{3}$ \\ ${ }^{1}$ Department of Physics and Materials Science, City University of Hong Kong, Tat Chee Avenue, \\ Kowloon Tong, Kowloon, Hong Kong \\ ${ }^{2}$ Faculty of Sciences, University of Kragujevac, 34000 Kragujevac, Yugoslavia \\ ${ }^{3}$ Faculty of Technology, University of Nis, Lescovac, Yugoslavia
}

Received 5 December 2000, in final form 10 May 2001

\begin{abstract}
The sensitivity to different relevant parameters of the absorbed fraction of alpha particles emitted from the ${ }^{222} \mathrm{Rn}$ chain in sensitive cells of the tracheo-bronchial tree have been investigated. The structure of the airway wall given by ICRP (ICRP66) has been adopted and employed in the present calculations. The source thickness (mucous gel and sol + cilia), target layer thickness and the depth of the sensitive layers have been varied within reasonable ranges around the default values recommended by ICRP66. The results have shown that the depth of the sensitive layers is the most important parameter in calculating the absorbed fraction. In addition, dose conversion coefficients were calculated and presented along with the absorbed fractions.
\end{abstract}

\section{Introduction}

Dose conversion factors for radon progeny in the human lung have been the subject of many calculations in the past (e.g. Harley and Pasternack 1982, NRC 1991, ICRP 1994 (ICRP66), Birchall and James 1994, Zock et al 1996, Porstendörfer and Reineking 1999, Marsh and Birchall 2000 etc). Dose conversion factor (DCF) is defined as the weighted effective dose in the human respiratory tract per unit exposure to radon progeny, and it is given in the unit $\mathrm{mSv} / \mathrm{WLM}$. Calculations of the DCF involve a few separate steps. The first is the determination of the absorbed fraction (AF) of alpha particles in the sensitive cells of the lung. The second one is calculation of the number $N_{\alpha}$ of alpha particles emitted in different regions of the lungs for the given exposure condition and time. This step involves determination of the deposition and clearance of radon progeny and their equilibrium activities in various regions of the lung. The third step calculates the product of $\mathrm{AF}$ and $N_{\alpha}$ to give the dose in different regions of the lung. The fourth step weights the doses obtained in the different regions to give a single value which is representative for the lung.

Determination of AF is an important step in DCF calculations, and it was previously determined by Haque (1967), NRC (1991), ICRP (1994) etc. Different authors used different physical quantities to express their results, but all of them are interrelated and can be 
recalculated from each other. For example NRC used dose conversion coefficient (DCC) in units of (nGy per disintegration $\mathrm{cm}^{-2}$ ), Haque (1967) used the 'number of equivalent alpha particles', while ICRP66 used the dimensionless quantity called absorbed fraction.

Calculation of AF can be performed using Monte Carlo methods as carried out by ICRP66. Another semi-analytical approach was described by Haque (1967), Al-affan and Haque (1989) and Crawford-Brown (1987). We have developed computer programs to calculate AF and DCC based on the semi-analytical approach. In contrast to the works of Haque (1967) and Crawford-Brown (1987), where the radioactivity was assumed to distribute on the mucus surface, we have considered volumetric distribution of activity in different cylindrical layers of airway wall tissue according to the ICRP66 model. This program has been tested against the results of ICRP (1994) and NRC (1991). Since good agreement was found we have used this program to study the dependence of $\mathrm{AF}$ on various relevant factors.

\section{Method}

The energy $E$ imparted to a small sphere with diameter $d_{\text {sp }}$ and a centre at a point $\mathrm{A}$ in the tissue is given by the following equation:

$$
E=\frac{2}{3} d_{\mathrm{sp}} \frac{\pi}{4} d_{\mathrm{sp}}^{2} N \iiint \frac{\rho \mathrm{d} \rho \mathrm{d} \varphi \mathrm{d} z}{4 \pi \mathrm{AC}^{2}} S\left(\mathrm{AC}_{\text {Tissue-eqv. }}\right)
$$

where $N$ is the volumetric activity (in $\mathrm{Bq} \mu \mathrm{m}^{-3}$ ), $\rho \mathrm{d} \rho \mathrm{d} \varphi \mathrm{d} z$ is an infinitesimally small volume element around point $\mathrm{C}$ in the cylindrical coordinate system, $\mathrm{AC}$ is a distance from the volume element to the small sphere centred at A where the imparted energy is calculated, $S$ is the stopping power of alpha particles after traversing the distance AC in tissue, $\pi d_{\mathrm{sp}}^{2} / 4$ is the cross-sectional area of the sphere and $2 d_{\mathrm{sp}} / 3$ is the average path length of particles through the sphere of diameter $d_{\mathrm{sp}}$. The limits of integration in equation (1) are determined by the range $R$ of alpha particles in tissue.

The integrals in the above expression cannot be solved analytically and numerical procedures should be applied. A computer program was developed to solve the integrals in equation (1). The variables $\rho, \varphi$ and $z$ were varied between the limits with the following steps: $\Delta \varphi=0.001$ degrees for the near wall and $\Delta \varphi=1$ degree for the far wall; $\Delta z=1 \mu \mathrm{m}$ and $\Delta \rho=1 \mu \mathrm{m}$.

The energy imparted to the sphere with diameter $d_{\mathrm{sp}}$ should be transformed to the absorbed dose by dividing the imparted energy by the mass of the sphere, which is proportional to $d_{\mathrm{sp}}^{3}$. Therefore, the absorbed dose is independent of the sphere diameter and additional assumptions about its diameter were not needed. For the purpose of calculation a sphere diameter of $1 \mu \mathrm{m}$ has been employed.

The stopping power of alpha particles in striated tissue was adopted from the ICRU49 report (ICRU 1993). The data given in ICRU (1993) were fitted by

$$
-\frac{\mathrm{d} E}{\mathrm{~d} x}=S(E)=\sum_{i=1}^{5} a_{i} E^{b_{i}} e^{c_{i} E}
$$

where $a_{i}$ and $b_{i}$ are constants.

Energy loss of alpha particles in air (in the case of the far wall) was taken into account as follows: the path length of an alpha particle in air was multiplied by the ratio, $T_{\text {air/tissue }}$, between the stopping powers in air and in tissue to give the tissue equivalent path length for those alpha particles passing through air.

The computer program was used to investigate the dependence of the $\mathrm{AF}$ of alpha particles in the sensitive cells of the human tracheo-bronchial tree on the thickness of different cell layers in the wall of airway tube. 
In the ICRP66 report, AF was defined as the average fraction of alpha particle energy absorbed in the sensitive cell layer. AF is given in ICRP66 as a function of alpha particle energy although in the radon chain only two energies are useful $\left(6 \mathrm{MeV}\right.$ emitted by ${ }^{218} \mathrm{Po}$ and 7.69 $\mathrm{MeV}$ from ${ }^{214} \mathrm{Po}$ ). In the ${ }^{220} \mathrm{Rn}$ (thoron) chain the energies $6.78,6.05,6.09$ and $8.78 \mathrm{MeV}$ will be needed. The dependence of AF on the thickness of relevant layers is also needed and it will be given in the present work.

The effects of some varying parameters on AF are quantified by a sensitivity factor $\mathrm{SF}$ defined as $\mathrm{SF}=\left|1-\mathrm{AF}_{\min } / \mathrm{AF}_{\max }\right|$ (Baixeras et al 1999), where $\mathrm{AF}_{\min }$ and $\mathrm{AF}_{\max }$ are minimum and maximum values of $\mathrm{AF}$ when a particular parameter is changed within a reasonable range while keeping all other parameters constant.

\section{Results}

In the present work we have adopted the model of airway wall and target-cell distribution given by NRC (1991) (pages 197 and 199) and ICRP66 (pages 15 and 17). Two models of the wall have been given for the bronchial (BB) and bronchiolar (bb) regions in these reports. The notations BB for bronchial and bb for bronchiolar region, introduced by ICRP66, are adopted in the present work. Two types of cell, viz., basal and secretory cells, are considered radiosensitive and critical targets for alpha particles. Two main differences can be found between the $\mathrm{BB}$ and $\mathrm{bb}$ regions, namely (i) the different thickness of the layers and (ii) the absence of basal cells in the bb region.

\subsection{Source thickness: mucous gel and sol + cilia thickness}

3.1.1. BB region. According to NRC (1991) and ICRP (1994) the wall of the airway tube in the BB region consists of the following layers: mucous gel $(5 \mu \mathrm{m})$, cilia + sol $(6 \mu \mathrm{m})$, fluid layer $(10 \mu \mathrm{m})$, secretory cell layer $(30 \mu \mathrm{m})$, basal cells $(15 \mu \mathrm{m})$ and basement membrane. The deepest part of the secretory-cell layer overlaps partially with the basal-cell layer. Since the range of alpha particles is very short in the tissue, the thickness of the layers are very important in calculating AF (and subsequently the effective dose).

Different authors used various assumptions about the thickness of the mucous layer. For example, Hofmann et al (2000) used $8 \mu \mathrm{m}$ as the mucus thickness, which is independent of the generation number of the airway tube, while Hui et al (1990) assumed a constant mucous blanket thickness of $15 \mu \mathrm{m}$.

We have varied the thickness of the mucous layer from 2 up to $12 \mu \mathrm{m}$ and calculated AF. The thickness of other layers have been kept constant. The results of these calculations are given in figure 1. The AF decreases with the mucous thickness because a larger mucous thickness will lead to (i) an increase in the average source-target distance; (ii) larger selfabsorption of alpha particles in the mucus, and (iii) larger shielding of the sensitive cells from the opposite side (far wall). The data for $6 \mathrm{MeV}$ alpha particles in basal cells are described by the right ordinate axis in figure 1. Being close to the end of the range for $6 \mathrm{MeV}$ alpha particles, the AF in the basal cells is much smaller than other AFs in the BB region. Furthermore, it is much more sensitive to the change of the mucous thickness. It decreases by a factor of 4.5 if the mucous gel thickness changes from 2 to $12 \mu \mathrm{m}$.

Figure 2 shows the results for the AF in the $\mathrm{BB}$ region when the thickness of the sol layer changes from 4 to $12 \mu \mathrm{m}$, while keeping the thickness of other layers constant. The AF decreases with the thickness of the sol layer for the same reasons as described previously. Being more sensitive to the changes of sol thickness, the AF in the basal cells for $6 \mathrm{MeV}$ alpha particles (described by the right axis of figure 2) decreases much more than others. 


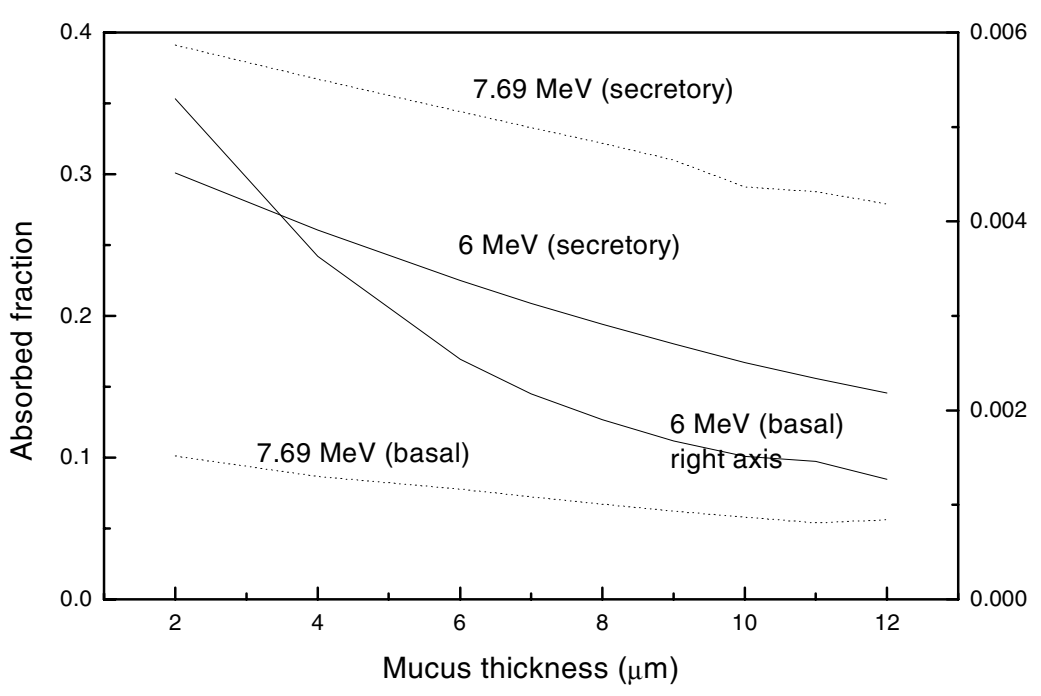

Figure 1. Absorbed fraction in basal and secretory cells in the BB (bronchial) region as a function of mucous gel thickness.

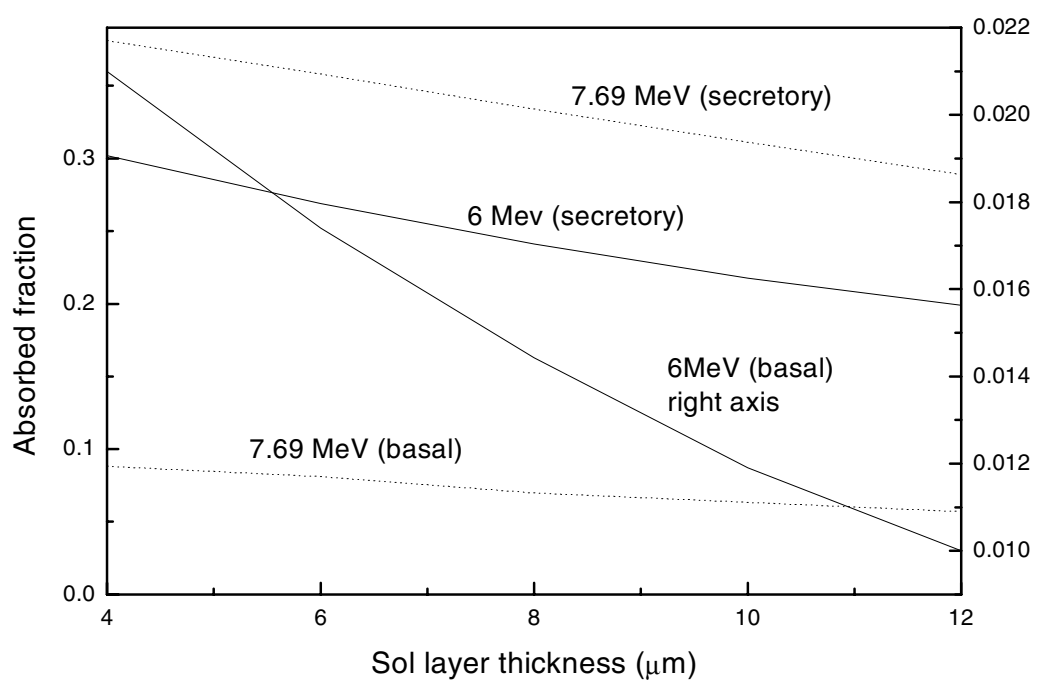

Figure 2. Absorbed fraction in basal and secretory cells in the BB (bronchial) region as a function of sol thickness.

3.1.2. bb region. The wall of the airway tube in the bb region consists of mucous gel $(2 \mu \mathrm{m})$, sol + cilia $(4 \mu \mathrm{m})$, a fluid epithelial layer without sensitive cells $(4 \mu \mathrm{m})$, secretory cells $(8 \mu \mathrm{m})$ and basement membrane. In this region only secretory cells are considered radiosensitive.

First, the thickness of mucous gel was varied from $1 \mu \mathrm{m}$ to $6 \mu \mathrm{m}$ while keeping all other thickness constant at their default values. Second, the thickness of the sol + cilia layer was changed from 2 to $8 \mu \mathrm{m}$ while keeping other parameters constant. The results of these calculations are given in figure 3. The curves for $6 \mathrm{MeV}$ alpha energy are well above those for $7.69 \mathrm{MeV}$, which can be explained by the decrease in the stopping power of alpha particles 


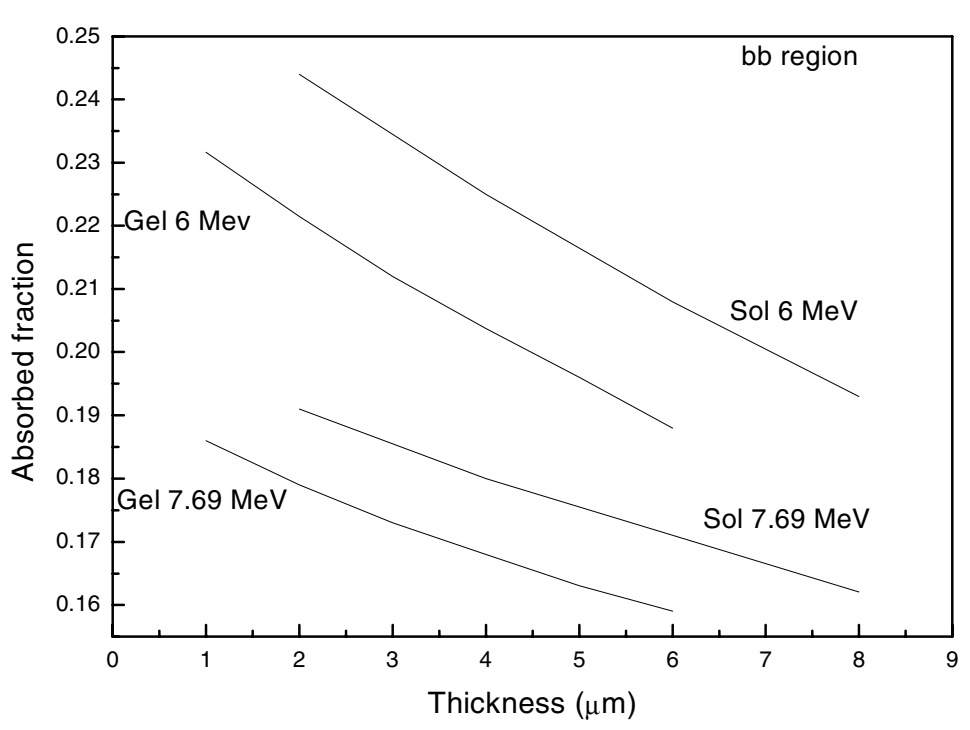

Figure 3. Absorbed fraction in secretory cells in the bb (bronchiolar) region as a function of source layer thickness.

with the increase in their energies. The target cells in the bb region are very close to the alpha source so alpha particles emitted with an initial energy of $6 \mathrm{MeV}$ enter the sensitive layer with smaller energy than those of $7.69 \mathrm{MeV}$. Furthermore, the AFs decrease with the increase in the layer thickness in the bb region.

\subsection{Thickness of sensitive layers}

According to ICRP66 the thickness of the secretory-cell layer is $30 \mu \mathrm{m}$ in the BB and $8 \mu \mathrm{m}$ in the bb region, and the thickness of the basal-cell layer is $15 \mu \mathrm{m}$ (these values are taken as the default lung-model parameters). These values were determined essentially by functional reasons rather than from body size or other lung-size parameters. Under such conditions there are no compelling reasons not to assume some wide range of values for these thicknesses. The thickness of secretory cells in BB was varied from $15 \mu \mathrm{m}$ up to $45 \mu \mathrm{m}$ (i.e. from $0.5 \times$ default thickness up to $1.5 \times$ default thickness). The other parameters, mucous gel and sol thickness and the depth where the sensitive layers begin, have been kept constant at their default values.

Figure 4 gives the results for AF and DCC (in nGy per disintegration $\mathrm{cm}^{-2}$ ) in secretory cells in the BB region as a function of the secretory-cell layer thickness. The source of alpha particles is the mucous gel layer ( $5 \mu \mathrm{m}$ thick) or the sol + cilia layer ( $6 \mu \mathrm{m}$ thick). There are four pairs of curves in figure 4, two for AF for 6 and 7.69 MeV, and the other two for DCC also for 6 and 7.69 MeV. The AF and DCC show opposite patterns in figure 4. The curves for AF increase and saturate - this saturation begins when the secretory cell is so thick that all alpha particles are completely stopped in it so that any further increase in the thickness of the layer does not enlarge AF. The lines for AF from 7.69 MeV did not saturate within the considered range of the secretory cell thickness. In contrast to AF, a thicker target layer means a larger mass of the sensitive tissue and the absorbed dose (in nGy) decreases, so that DCCs diminish monotonically.

Figure 5 gives the results for AF and DCC in basal cells in the BB region, when the alpha particles source are in the mucous gel or in sol + cilia layers. The thickness of basal-cell 


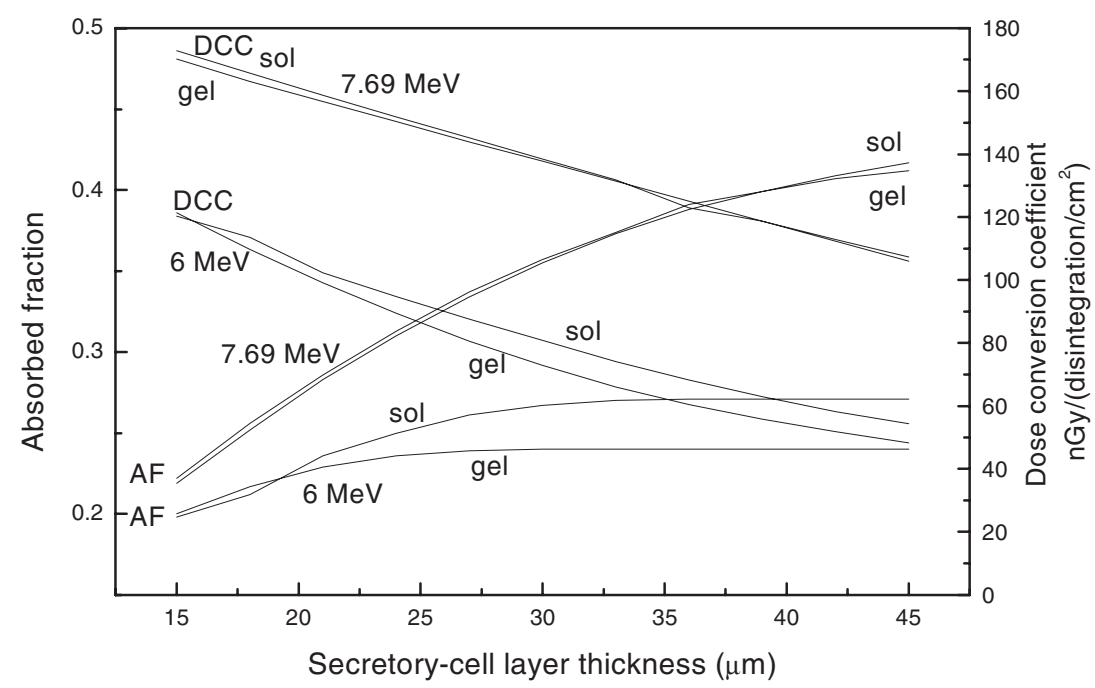

Figure 4. Absorbed fraction and dose conversion coefficient for secretory cells in the BB region as a function of secretory-cell layer thickness. AF values are described by the left-hand axis, and DCC values by the right-hand axis. The sources are the mucous gel and sol+cilia in the BB region.

layer has been varied between $7 \mu \mathrm{m}$ and $22 \mu \mathrm{m}(\approx 15 \pm 0.5 \times 15)$. It is anticipated that the thickness of the basal-cells layer falls within these limits. The AF for $6 \mathrm{MeV}$ alpha particles is independent of the basal-cell layer thickness because the alpha particles are stopped in the target layer, which explains why the lines representing these data are horizontal in figure 5 . The AF for $7.69 \mathrm{MeV}$ alpha particles increases continuously in the range and does not saturate. Data for DCC are described by the right-hand ordinate axis (notice the logarithmic scale). DCC decreases with the basal-cell layer thickness because of the larger target mass layer; the values for $7.69 \mathrm{MeV}$ alpha particles decrease from 80 to $50 \mathrm{nGy}$ (disintegration $\mathrm{cm}^{-2}$ ) if the basal-cell layer thickness is increased from 8 to $22 \mu \mathrm{m}$. The $\mathrm{DCC}_{\mathrm{gel}}$ for $6 \mathrm{MeV}$ alpha particles decreases even more, from 2.9 to $1 \mathrm{nGy}$ (disintegration $\mathrm{cm}^{-2}$ ).

Figure 5 also shows the results for basal cells, if the alpha-particle source is in the sol+cilia layer. The main differences between the results for gel and sol are numerical values for $6 \mathrm{MeV}$ alpha particles, e.g. $\mathrm{AF}_{\text {gel }}=0.00256$ and $\mathrm{AF}_{\text {sol }}=0.0168$. The difference in DCCs are similar and are five to eight times larger for the source in sol than in mucus. The values for 7.69 MeV alpha particles are close for the two different sources.

Figure 6 gives the results for AF and DCCs in secretory cells in the bb region as a function of the secretory-cell layer thickness. The AFs increase almost linearly because the target layer is too thin and most of the alpha particles are not stopped in it. The AFs for $6 \mathrm{MeV}$ alpha particles are larger than those for $7.69 \mathrm{MeV}$ ones. The DCCs decrease also linearly with the target thickness, and they are significantly larger than the DCC values for secretory cells in the $\mathrm{BB}$ region (cf results described by the right-hand ordinate axis in figure 4 ).

\subsection{Depth of sensitive cells}

In the previous section, we have varied the thickness of sensitive layers but the depth where the sensitive layers begin was kept constant. In this section, the thickness of the sensitive layers were kept constant but the starting depth was varied. 


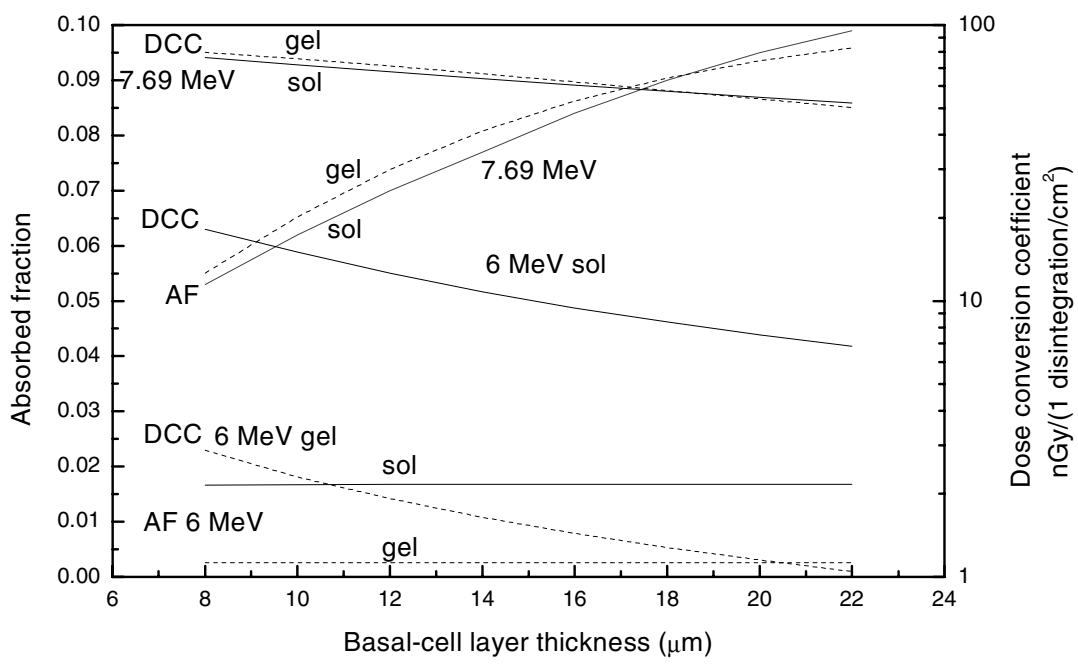

Figure 5. Absorbed fraction and dose conversion coefficient in basal cells in the BB region as a function of basal-cell layer thickness. AF values are described by the left-hand axis, and DCC values by the right-hand axis. The sources are the mucous gel and cilia + sol layer in the BB region. (Broken line, gel source; solid line, sol source.)

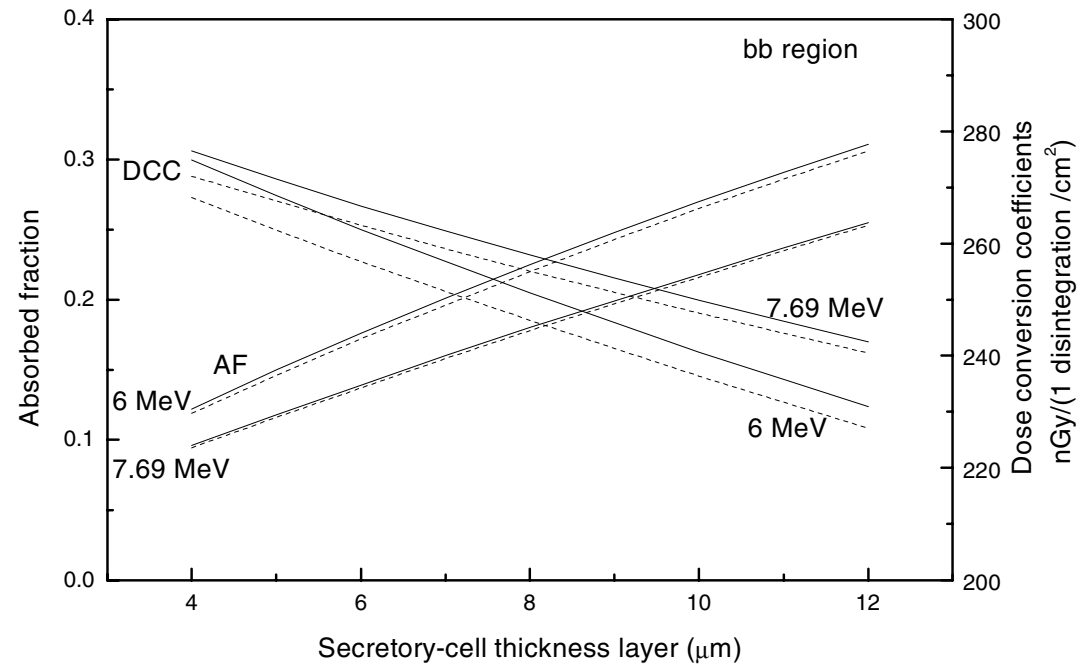

Figure 6. Absorbed fraction and dose conversion coefficient in secretory cells in the bb region as a function of secretory-cell layer thickness. AF values are described by the left-hand axis, and DCC values by the right-hand axis. The sources are mucous gel in the bb region (broken lines) and sol + cilia in the bb region (solid lines).

3.3.1. BB region. According to ICRP66 the secretory-cell layer begins at $21 \mu \mathrm{m}$ and ends at $51 \mu \mathrm{m}$ below the top of the mucus. The shallowest depth of the secretory-cell layer cannot be smaller than $11 \mu \mathrm{m}$, and the maximum depth is taken as $21+21 \times 0.5=32 \mu \mathrm{m}$. Therefore, we varied the starting depth of the secretory-cell layer between 11 and $32 \mu \mathrm{m}$ and calculated AFs and DCCs for the two alpha-particle sources (in mucus and sol + cilia). Figure 7 shows 


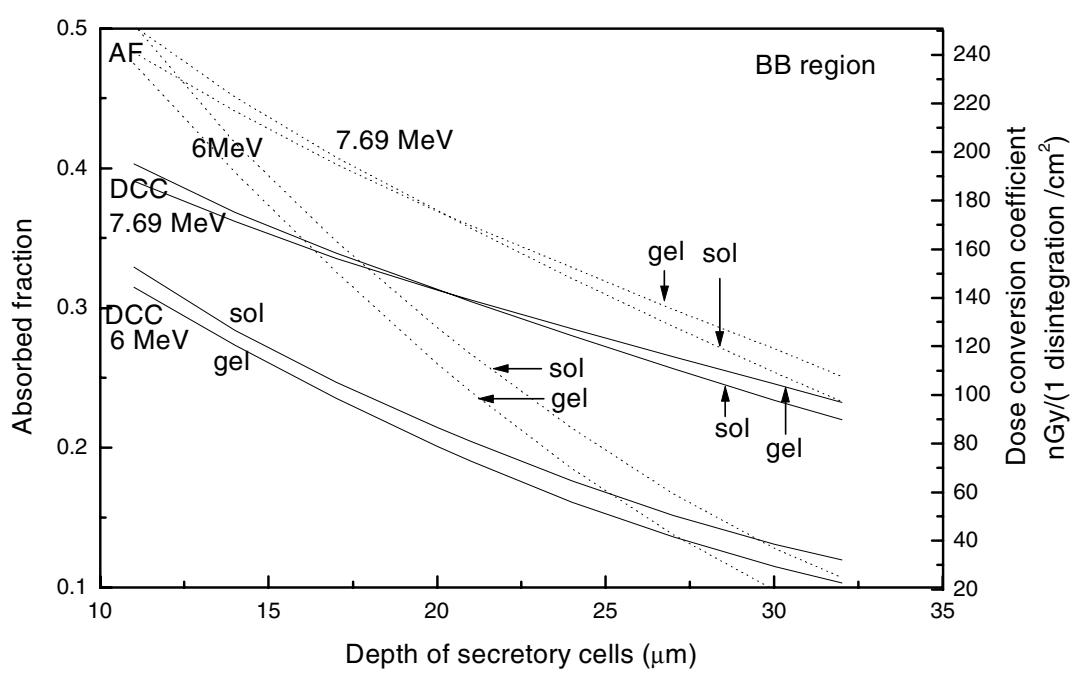

Figure 7. Absorbed fraction (described by the left-hand ordinate and represented by dotted lines) and dose conversion coefficient (described by the right-hand ordinate and represented by solid lines) in secretory cells in the BB region as a function of the depth of the secretory-cell layer.

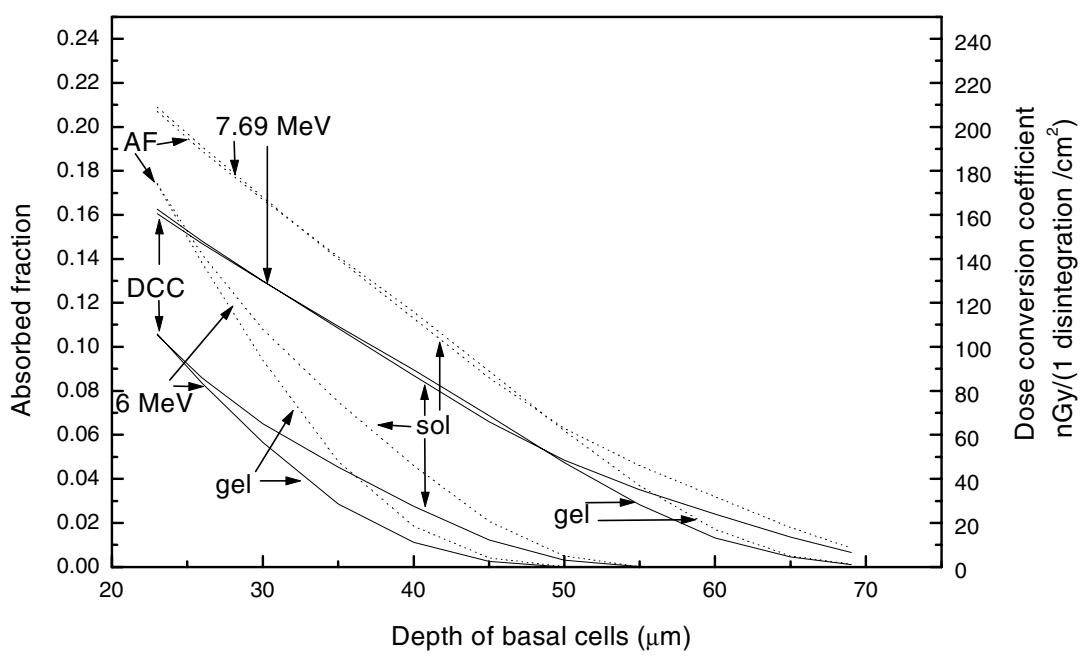

Figure 8. Absorbed fraction (described by the left-hand ordinate and represented by dotted lines) and dose conversion coefficient (described by the right-hand ordinate and represented by solid lines) in basal cells in the BB region as a function of the depth of the basal-cell layer.

the results. It can be seen that all AFs and DCCs decrease with the starting depth of the secretory-cell layer.

Figure 8 gives the results for AFs and DCCs in basal cells. The starting depth of the basal-cell layer was varied in the range between $23 \mu \mathrm{m}$ and $69 \mu \mathrm{m}(46 \pm 0.5 \times 46 \mu \mathrm{m})$. The results are also given for the source in mucous gel and in the sol + cilia layer. The values for basal cells decrease more that those for secretory cells. In some cases (curves for $6 \mathrm{MeV}$ ), AF as well as DCC drop to zero. 


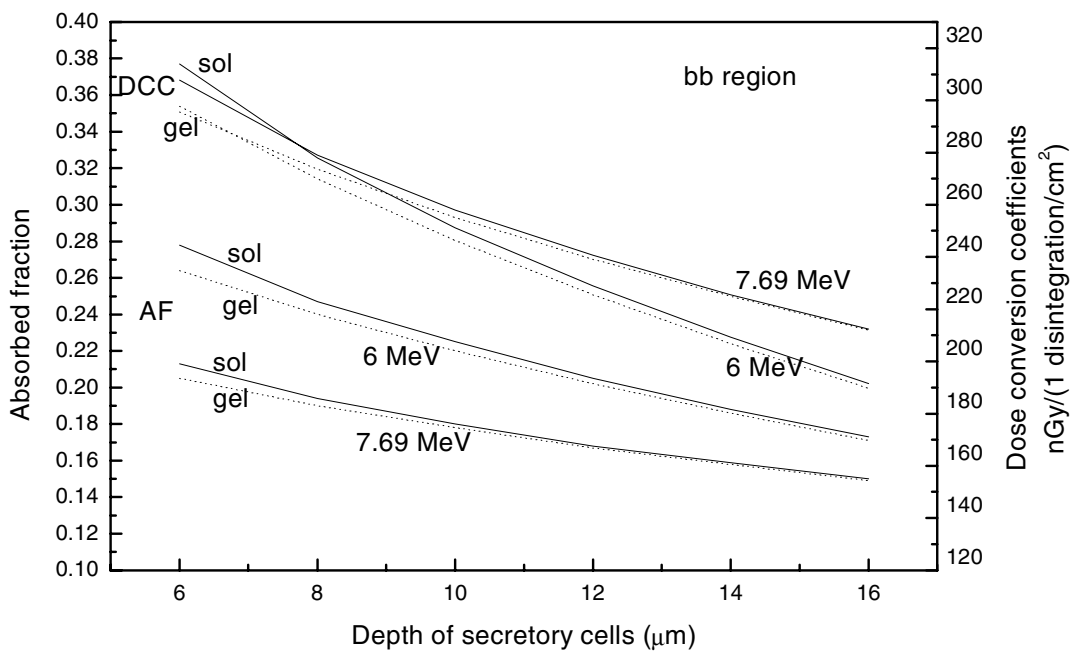

Figure 9. Absorbed fraction (described by the left-hand ordinate) and dose conversion coefficient (described by the right-hand ordinate) in secretory cells in the bb region as a function of the depth of the secretory-cell layer. The gel source is represented by dotted lines and the sol + cilia source by solid lines.

3.3.2. $\quad$ b $b$ region. The starting depth of secretory cells in the bb region has been changed from 6 to $16 \mu \mathrm{m}$ while keeping other parameters constant, including the layer thickness. The results are given in figure 9. The $\mathrm{AF}$ is larger for $6 \mathrm{MeV}$ alpha particles than that for $7.69 \mathrm{MeV}$ alpha particles. All AFs and DCCs decrease with the starting depth of secretory cells.

\subsection{Sensitivity factors}

Sensitivity factors of AF have been calculated for the source thickness, target layer thickness and depth of sensitive layer. The results are given in table 1 . We have considered three groups of sensitivities: low, where the sensitivity factor is less than 0.33 ; medium sensitivity between 0.33 and 0.66 (in italic in table 1) and high sensitivity (shown in bold in table 1).

The $\mathrm{AF}$ of $6 \mathrm{MeV}$ alpha particles is more sensitive to the variation of different parameters than that for $7.69 \mathrm{MeV}$ alpha particles. Source thickness is not a critical parameter in determining AF except in the case of gel source of basal cells. The most critical parameter in determining of $\mathrm{AF}$ is the starting depth of the sensitive layer.

\subsection{Scaling}

The above calculations were performed by varying one thickness, while keeping all others constant. However, all the thickness can be different at the same time from default ICRP values. One can assume that all the thicknesses 'behave' in a similar way; i.e. if one thickness is larger than the ICRP default, others will also be larger. This means that scaling of the thickness of all layers can be applied in calculating AF. This has been performed and the results are given in tables 2 and 3 . Four scaling factors have been studied, namely, $k=0.5$, $0.75,1.25$ and 1.5. Tables 2 and 3 show significant decreases in all AFs with the scaling factor. If AF is needed for some other scaling factors between those presented here, linear interpolation can be applied. 
Table 1. Sensitivity factors for the absorbed fraction. The sensitivity factors are categorized into low $(<0.33$ ); medium (between 0.33 and 0.66 ; figures in italic) and high $(>0.66$; figures in bold).

\begin{tabular}{|c|c|c|c|c|c|c|}
\hline \multirow[b]{2}{*}{$\begin{array}{l}\text { Varied } \\
\text { parameter }\end{array}$} & \multirow[b]{2}{*}{$\begin{array}{l}\text { Limits } \\
(\mu \mathrm{m})\end{array}$} & \multirow{2}{*}{$\begin{array}{l}\text { ICRP66 } \\
\text { default value } \\
(\mu \mathrm{m})\end{array}$} & \multirow{2}{*}{$\begin{array}{l}\text { Region } \\
\text { and } \\
\text { source }\end{array}$} & \multirow[b]{2}{*}{ Target cells } & \multicolumn{2}{|c|}{ Initial energy } \\
\hline & & & & & $\begin{array}{l}6 \mathrm{MeV} \\
\text { Sensitivity }\end{array}$ & $\begin{array}{l}7.69 \mathrm{MeV} \\
\text { Sensitivity }\end{array}$ \\
\hline Source & $2-12$ & 5 & BB-gel & Secretory & 0.52 & 0.28 \\
\hline thickness & $2-12$ & 5 & BB-gel & Basal & 0.76 & 0.44 \\
\hline gel & $1-6$ & 2 & bb-gel & Secretory & 0.18 & 0.14 \\
\hline Source & $4-12$ & 6 & BB-sol & Secretory & 0.34 & 0.24 \\
\hline thickness & $4-12$ & 6 & BB-sol & Basal & 0.52 & 0.33 \\
\hline sol & $2-10$ & 4 & bb-sol & Secretory & 0.21 & 0.15 \\
\hline Target & $15-45$ & 30 & BB-gel & Secretory & 0.17 & 0.47 \\
\hline layer & $8-22$ & 15 & BB-gel & Basal & 0 & 0.43 \\
\hline thickness & $4-12$ & 8 & bb-gel & Secretory & 0.61 & 0.62 \\
\hline Target & $15-45$ & 30 & BB-sol & Secretory & 0.27 & 0.47 \\
\hline layer & $8-22$ & 15 & BB-sol & Basal & 0.05 & 0.46 \\
\hline thickness & $4-12$ & 8 & bb-sol & Secretory & 0.61 & 0.62 \\
\hline Depth of & $11-32$ & 21 & BB-gel & Secretory & 0.84 & 0.48 \\
\hline sensitive & $23-69$ & 46 & BB-gel & Basal & 1 & 0.99 \\
\hline layer & $6-16$ & 10 & bb-gel & Secretory & 0.35 & 0.27 \\
\hline Depth of & $11-32$ & 21 & BB-sol & Secretory & 0.78 & 0.54 \\
\hline sensitive & $23-69$ & 46 & BB-sol & Basal & 1 & 0.95 \\
\hline layer & $6-16$ & 10 & bb-sol & Secretory & 0.62 & 0.29 \\
\hline
\end{tabular}

Table 2. Absorbed fractions in basal and secretory cells in the BB region for the scaled thickness. All thickness are multiplied by the scaling factor $k$. ICRP66 values $(k=1)$ are given in brackets for comparison.

\begin{tabular}{|c|c|c|c|c|c|c|c|c|}
\hline \multirow[b]{3}{*}{$k$} & \multicolumn{4}{|c|}{ Secretory cells (BB region) } & \multicolumn{4}{|c|}{ Basal cells (BB region) } \\
\hline & \multicolumn{2}{|c|}{$6 \mathrm{MeV}$} & \multicolumn{2}{|c|}{$7.69 \mathrm{MeV}$} & \multicolumn{2}{|c|}{$6 \mathrm{MeV}$} & \multicolumn{2}{|c|}{$7.69 \mathrm{MeV}$} \\
\hline & Mucus & Gel + cilia & Mucus & Gel + cilia & Mucus & Gel + cilia & Mucus & Gel + cilia \\
\hline 0.5 & 0.340 & 0.343 & 0.291 & 0.294 & 0.106 & 0.106 & 0.106 & 0.106 \\
\hline 0.75 & 0.331 & 0.331 & 0.334 & 0.338 & 0.05451 & 0.062 & 0.113 & 0.113 \\
\hline 1 & 0.256 & 0.278 & 0.356 & 0.359 & $5.2 \times 10^{-3}$ & 0.0221 & 0.09112 & 0.08755 \\
\hline (ICRP66) & $(0.249)$ & $(0.272)$ & $(0.353)$ & $(0.355)$ & $\left(5.06 \times 10^{-3}\right)$ & $(0.0217)$ & $(0.0893)$ & $(0.0857)$ \\
\hline 1.25 & 0.136 & 0.207 & 0.328 & 0.330 & 0 & $9.35 \times 10^{-4}$ & 0.0359 & 0.05209 \\
\hline 1.5 & 0.098 & 0.159 & 0.2690 & 0.289 & 0 & 0 & 0.00556 & 0.023491 \\
\hline
\end{tabular}

\section{Conclusions}

The most important parameter in determining AF and DCC is the starting depth of the sensitive cells. This parameter can significantly affect AF and subsequently the absorbed and effective doses. Other studied parameters, such as the source thickness and target layer thickness have smaller, but not negligibly, influence on AF.

DCCs are also calculated in the present work. In some cases DCCs behave in an opposite way from AF, which is due to the fact that the target mass is used for DCC calculations but not in determining AFs. 
Table 3. Absorbed fractions in secretory cells in the bb region for the scaled thickness. ICRP66 values $(k=1)$ are given in brackets for comparison.

\begin{tabular}{|c|c|c|c|c|}
\hline \multirow[b]{3}{*}{$k$} & \multicolumn{4}{|c|}{ Secretory cells (bb region) } \\
\hline & \multicolumn{2}{|c|}{$6 \mathrm{MeV}$} & \multicolumn{2}{|c|}{$7.69 \mathrm{MeV}$} \\
\hline & Mucus & Gel + cilia & Mucus & Gel + cilia \\
\hline 0.5 & 0.151 & 0.152 & 0.114 & 0.113 \\
\hline 0.75 & 0.189 & 0.191 & 0.147 & 0.147 \\
\hline 1 & 0.218 & 0.221 & 0.176 & 0.176 \\
\hline (ICRP66) & $(0.214)$ & $(0.217)$ & $(0.172)$ & $(0.173)$ \\
\hline 1.25 & 0.236 & 0.240 & 0.197 & 0.198 \\
\hline 1.5 & 0.248 & 0.252 & 0.217 & 0.219 \\
\hline
\end{tabular}

The results presented here can be useful for calculating the absorbed and effective doses from the short lived radon progeny when the thicknesses of various layers in the airway wall are different from the ICRP66 default values. Examples of applications are the dose calculations for various ethnic groups different from Caucasian man, for children and infants, for people suffering from lung illnesses, or for other cases when the airway wall is changed (such as due to smoking).

\section{Acknowledgment}

The present research is supported by the CERG grant CityU1004/99P from the Research Grant Council of Hong Kong.

\section{References}

Al-affan I A M and Haque A K M M 1989 Local energy deposited for alpha particles emitted from inhaled radon daughters Phys. Med. Biol. 34 97-105

Baixeras C, Amgarou K, Font L and Domingo C 1999 Long-term radon levels and equilibrium factor in some Spanish workplaces measured with a passive integrating detector Radiat. Prot. Dosim. 85 233-6

Birchall A and James A C 1994 Uncertainty analysis of the effective dose per unit exposure from radon progeny and implications for ICRP risk-weighting factors Radiat. Prot. Dosim. 53 133-40

Crawford-Brown D J 1987 Dosimetry Environmental Radon. (Environmental Science Research Volume 35) ed C H Cothern and J E Smith Jr (New York: Plenum) pp 192-99

Haque A K M M 1967 Energy expended by alpha particles in lung tissue II. A computer method of calculation $B r . J$. Appl. Phys. 18 657-62

Harley N H and Pasternack B S 1982 Environmental radon daughter alpha dose factors in a five-lobed human lung Health Phys. 42 789-99

Hofmann W, Menache M G, Crawford-Brown D J, Caswell R S and Karam L 2000 Modeling energy deposition and cellular radiation effects in human bronchiolar epithelium by radon progeny alpha particles Health Phys. 78 377-93

Hui T E, Poston J W and Fisher D R 1990 The microdosimetry of radon decay products in the respiratory tract Radiat. Prot. Dosim. 31 405-11

International Commission of Radiation Units and Measurements (ICRU) 1993 Stopping powers and ranges for protons and alpha particles ICRU Report 49

International Commission on Radiological Protection (ICRP) 1994 Human Respiratory Tract Model for Radiological Protection ICRP66, vol 24 (Oxford: Pergamon)

Marsh J W and Birchall A 2000 Sensitivity analysis of the weighted equivalent dose per unit exposure from radon progeny Radiat. Prot. Dosim. 87 167-78 
National Research Council (NRC) 1991 Comparative Dosimetry of Radon in Mines and Homes. Panel on Dosimetric Assumption Affecting the Application of Radon Risk Estimates (Washington, DC: NRC, National Academy Press)

Porstendörfer J and Reineking A 1999 Radon: characteristics in air and dose conversion factors Health Phys. 76 $300-5$

Zock C, Porstendörfer J and Reineking A 1996 The influence of biological and aerosol parameters of inhaled short-lived radon decay products on human lung dose Radiat. Prot. Dosim. 63 197-206 